\title{
Palliative care for patients with Parkinson's disease: an interdisciplinary review and next step model
}

This article was published in the following Dove Press journal:

Journal of Parkinsonism and Restless Legs Syndrome

20 February 2017

Number of times this article has been viewed

\author{
Kimmy G Su' \\ Julie H Carter ${ }^{\prime}$ \\ Keiran K Tuck ${ }^{2}$ \\ Tony Borcich ${ }^{3}$ \\ Linda A Bryans ${ }^{4}$ \\ Lisa L Mann' \\ Jennifer $L$ Wilhelm ${ }^{5}$ \\ Erik K Fromme ${ }^{6}$ \\ 'Department of Neurology, Oregon \\ Health \& Science University, ${ }^{2}$ The Oregon \\ Clinic-Neurology, ${ }^{3}$ Parkinson's Resources \\ of Oregon, ${ }^{4}$ Northwest Clinic for Voice and \\ Swallowing, Oregon Health and Science \\ University, ${ }^{5}$ Rehabilitation Services, Oregon \\ Health \& Science University, ${ }^{6}$ Palliative Care \\ Section, OHSU Knight Cancer Institute, \\ Portland, OR, USA
}

\section{Video abstract}

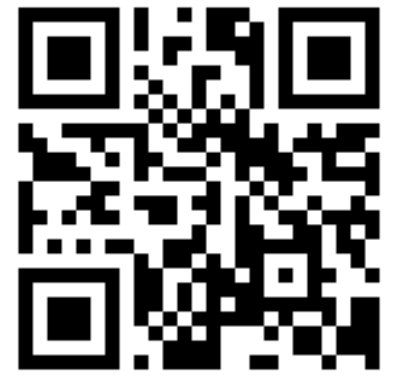

Point your SmartPhone at the code above. If you have a QR code reader the video abstract will appear. Or use: http://youtu.be/Paju6e0702Y

Correspondence: Erik K Fromme Oregon Health \& Science University, Palliative Care Section, L586, 3I8I SW Sam Jackson Park Road, Portland, OR 97239-3098, USA

Tel +l 5034943152

Fax +I 5034943257

Email frommee@ohsu.edu
Abstract: Late stage Parkinson's and Parkinson-plus patients have increased needs beyond motor symptom management that cannot be fully addressed in a typical neurology clinic visit. Complicating matters are the concurrent increasing emotional and physical demands on caregivers, which, if addressed, further stretch clinic time constraints. The complex and extensive patient and caregiver needs warrant a dedicated clinic to provide the necessary interdisciplinary care. In contrast to a typical model where the neurology clinician refers the patient to various ancillary treatment groups resulting in multiple separate clinic visits, the interdisciplinary model supports direct communication between the different disciplines during the clinic visit, allowing for a more coordinated response that takes into account multiple perspectives. Such an interdisciplinary model has been utilized in neurologic disorders with complex end-stage disease needs, such as amyotrophic lateral sclerosis with notable improvement in quality of life and survival. The Oregon Health \& Science University Parkinson Center and Movement Disorders Clinic has developed an interdisciplinary clinic called Next Step composed of neurology clinicians, a physical therapist, a speech pathologist, a social worker, and a nursing coordinator. The clinic focuses on palliative care issues, including complex late stage motor symptoms, nonmotor symptoms, and quality of life goals of both the patient and caregiver(s). This article describes the Next Step clinic structure and processes, while reviewing the literature and incorporating clinical expertise from the perspective of each discipline.

Keywords: palliative care, Parkinson's disease, caregiver burden, interdisciplinary team, latestage Parkinson's, quality of life

\section{Introduction}

Parkinson's disease (PD) and Parkinson's-plus diseases (PD+) are chronic, progressive, and incurable neurodegenerative diseases that currently affect more than 10 million people worldwide. This number is only to increase as the world population ages. ${ }^{1}$ In addition to the increasing number of PD patients to be seen in neurology clinics for specialized care, the patients themselves also develop increasing needs at later stages of disease. Initially, patient care is usually focused on motor symptoms, including tremors, postural instability, gait, and rigidity. However, with disease progression, patient needs extend beyond motor symptoms and encompass a wide range of nonmotor symptoms including autonomic dysfunction, cognitive complaints, mood issues, pain, and psychosis. Longitudinal studies for PD patients have shown a notable increase in symptom burden and worsening quality of life at later stages of disease. Higginson et $\mathrm{al}^{2}$ showed that $>80 \%$ of PD patients developed four symptoms of pain, difficulty using legs, fatigue, and sleepiness, and $>50 \%$ of patients developed nine symptoms 
of communication problems, oral problems, such as drooling, use of upper extremities, spasms/stiffness, sleep issues, constipation, bladder issues, swallowing issues, and shortness of breath over the course of a year.

As symptom burden increases in patients with late stage PD, so does caregiver burden or strain. The accumulation of caregiver strain at later stages of disease includes "worry, tension, frustration from communication problems, direct care, role conflict, and global strain," as well as "lack of resources, economic burden, feelings of being manipulated, and mismatched expectations." 3

The World Health Organization (WHO) defines palliative care as

"an approach that improves the quality of life of patients and their families facing the problem associated with lifethreatening illness, through the prevention and relief of suffering by means of early identification and impeccable assessment and treatment of pain and other problems, physical, psychosocial and spiritual."

As per the WHO definition, this palliative care approach, which is often team-based, not only can enhance quality of life but also can positively influence the course of illness. As PD progresses, the burgeoning palliative care needs of the patient and caregiver can be overwhelming for neurology clinicians to address within the time constraints of a typical clinic visit. Furthermore, as patients near the end of their lives, they and their family members may wish to initiate goals of care discussions and revisit advanced care planning and financial planning for the future. These are time intensive discussions that cannot be rushed. In a typical model, the neurology clinician can refer the patient to various ancillary treatment groups (physical therapy, speech pathology, social work, etc.) for further management; however, this results in many more clinic visits that can be difficult for patient and caregiver to attend due to transportation and mobility constraints. Furthermore, this model limits communication between the different care providers, and care is more likely to be disjointed (Figure 1A).

Increasing patient and caregiver needs with late stage disease is not unique to PD. To address the issue of complex patient and caregiver management, we can look to other neurologic disorders with similar late stage symptom burden, such as amyotrophic lateral sclerosis (ALS) and muscular dystrophy. These diseases have utilized multidisciplinary/ interdisciplinary models to facilitate care with noted success. For instance, studies have shown that multidisciplinary clinic models composed of neurology clinicians, physical, occupational and speech therapists, pulmonologist, nutritionist,
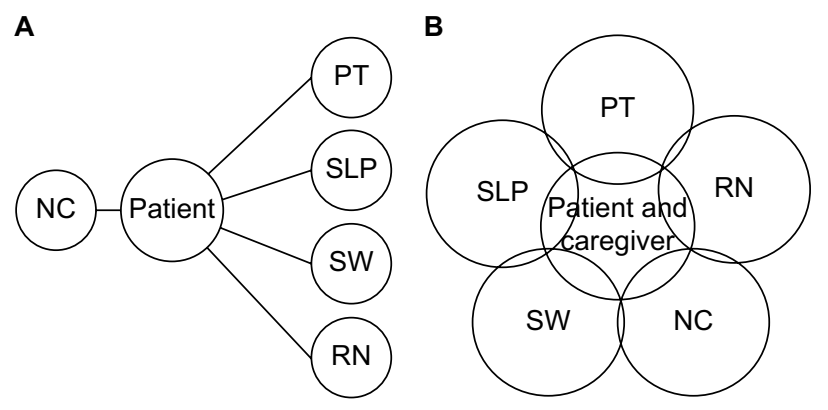

Figure I Comparison of traditional and interdisciplinary clinic models.

Notes: (A) Traditional clinic model: The neurology clinician, after seeing the patient in clinic, places referrals to PT, SLP, SW, and RN. The patient then sees each discipline at a separate visit. (B) Interdisciplinary clinic model: Both patient and caregiver are seen within the same visit by the different disciplines. Close communication is maintained between disciplines during the visit, and a comprehensive care plan is ultimately developed following dedicated discussions.

Abbreviations: NC, neurology clinician; PT, physical therapist; RN, nursing coordinator; SLP, speech language pathologist; SW, social worker.

and social worker (SW) have actually increased survival in patients with ALS by several months. ${ }^{5}$ While unclear about the exact mechanism for improved survival, the authors hypothesize that the multidisciplinary team providing more comprehensive care, more frequent interactions, and more counseling may be responsible.

In 2014, we started the Next Step Clinic at Oregon Health \& Science University to better care for our PD and PD+ patients. The clinic utilizes the interdisciplinary approach toward care of late stage PD patients, incorporating the skills of neurology clinicians, a physical therapist (PT), a speech language pathologist (SLP), a SW, and a nursing coordinator $(\mathrm{RN})$ within the same visit (Figure 1B). In addition to providing a range of expertise, the clinic also shifts priority of care from restorative management to quality of life goals as directed by the patient and family. In the following sections, clinic logistics will be discussed, including the patient referral process, the team professionals involved, and the flow of the clinic day. This section will be followed by a review from the perspective of each discipline involved in the clinic, an overview of the symptoms that are typically encountered, and the associated treatment options. The reviews synthesize published literature, when available, with each professional's clinical experience and expertise.

\section{Clinic logistics/operation}

\section{Referral criteria}

Referral criteria include debilitating motor symptoms, complex nonmotor symptoms (e.g., pain, cognitive issues, depression, and autonomic dysfunction), caregiver strain, care goals and end-of-life document discussions, and help with transitions of care (e.g., moving into a long-term care facility). 


\section{Pre-assessment}

Prior to their appointment, patients are mailed a set of questionnaires along with an information package explaining the purpose of the clinic and encouraging caregiver(s) to attend. Patients are asked to complete the Parkinson's Disease Questionnaire-39, Edmonton Symptom Assessment Scale-PD, and Center for Epidemiologic Studies Depression Scale (CES-D). Caregivers are asked to complete the CES-D and Modified Caregiver Strain Index (MCSI). We have also found it helpful to ask patients to list the three things that are most important to their quality of life.

\section{Team health care professionals}

Our team consists of neurology clinicians (medical doctor [MD], nurse practitioner [NP], or physician assistant [PA]), a PT, a SLP, a SW, and a RN.

\section{Clinic schedule}

Next Step Clinic runs twice a month for half a day, with the aim to see three new patients and two follow-up patients each half day. The clinic schedule can be seen in Table 1. New patients are seen by all team members. Follow-up patients are usually only seen by the neurology clinicians; the other team members may see them on an as needed basis.

Clinic starts with a 30-minute preclinic team meeting to discuss the day's patients, review completed questionnaires, and assign schedules. After this meeting, the first round of visits begins for the new patients, lasting for $\sim 45$ minutes. The entire team then meets for 15 minutes to discuss the new patients. This mid-clinic team meeting provides guidance for the next team of providers and prevents the patients from having to repeat themselves. The teams continue to rotate through the new patients after the meeting, and one clinician begins seeing the follow-up patients. After another 45-minute visit, there is a post-clinic team meeting during which a final treatment plan is created involving the input of all team members. One of the benefits of this interdisciplinary approach is that it allows the team to maximize nonpharmacologic approaches to symptom management in this frail population. This jointly derived end-of-visit plan is then relayed in verbal and written form to the new patients by the clinician, including any recommended referrals or medication prescriptions. Throughout the day, patients and their caregivers remain in the same room while the team members move between rooms, thus reducing burden for less mobile patients.

\section{Narrative review of palliative care needs in PD from the perspectives of health care professionals involved in the next step clinic: Neurology clinician (MD, NP, or PA)}

The neurology clinician's role shifts in this particular interdisciplinary clinic to addressing the nonmotor symptoms prominent in late stage PD. In depth review of these symptoms and their management can be found elsewhere. ${ }^{6-8}$ Here, we will briefly highlight some of the symptoms that particularly benefit from a palliative interdisciplinary approach.

\section{Pain}

Pain is a common, but under-recognized symptom in PD. Up to $85 \%$ of people with $\mathrm{PD}$ experience pain, and up to $63 \%$ of that pain is directly attributable to PD. ${ }^{9-11}$ Furthermore, many patients with PD (25\% in the early stage and $16 \%$ in the late stage) consider pain one of their top three most bothersome symptoms, significantly affecting their quality of life. ${ }^{12}$ Patients with PD-related pain score lower on measures of motor function, mental health, depression, social functioning, and general health. ${ }^{13}$ Furthermore, caregivers

Table I Template of next step clinic schedule

\begin{tabular}{|c|c|c|c|c|c|}
\hline \multirow[t]{2}{*}{ Time } & \multicolumn{5}{|l|}{ Patient } \\
\hline & New I & New 2 & New 3 & Return I & Return 2 \\
\hline $8: 30-9: 00$ & \multicolumn{5}{|c|}{ Preclinic meeting } \\
\hline 9:00-9:45 & $\mathrm{NCI}+\mathrm{SW}$ & NC 2 & $\mathrm{PT}+\mathrm{SLP}$ & & \\
\hline $9: 45-10: 00$ & \multicolumn{5}{|c|}{ Mid-clinic team meeting } \\
\hline $10: 00-10: 20$ & PT & $S L P+S W$ & $\mathrm{NC} \mathrm{I}$ & NC 2 & \\
\hline $10: 20-10: 45$ & SLP & PT & $\mathrm{NCI}+\mathrm{SW}$ & & NC 2 \\
\hline $10: 45-11: 15$ & \multicolumn{5}{|c|}{ Post-clinic meeting } \\
\hline $\mid 1: 15-11: 30$ & \multicolumn{5}{|c|}{ End-of-visit care plan discussion with either NC I or NC 2} \\
\hline
\end{tabular}

Notes: Template of schedule used in the Next Step Clinic that incorporates seeing three new patients and two return patients within a half-day. The patient and caregivers remain in the same room whereas the health care professionals rotate between rooms. There are several scheduled team meetings that occur prior to, during, and at the end of the clinic visit.

Abbreviations: NC, neurology clinician; PT, physical therapist; SLP, speech language pathologist; SW, social worker. 
of PD patients describe end-of-life pain as common and poorly managed. ${ }^{14}$

Unfortunately, there are no Food and Drug Administration-approved medications, class A randomized control trials or widely accepted guidelines for pain management in PD. Currently, pain management is largely determined by the underlying etiology of the pain symptoms. A review by Ford divides Parkinsonian pain into five categories (musculoskeletal, radicular/neuropathic, dystonic, central, and akathetic), and discusses recommended treatments associated with each type of pain. ${ }^{15}$ Musculoskeletal pain can be treated with physical therapy and simple analgesics, such as acetaminophen or nonsteroidal anti-inflammatory drugs. Dystonic pain can be treated with the adjustment of dopaminergic medications and/or botulinum toxin. Radicular and neuropathic pain can be treated with centrally acting pain medications, such as gabapentin or duloxetine. Central pain syndromes are difficult to treat, but may respond to adjustment of dopaminergic medications or centrally acting pain medications, such as gabapentin or duloxetine. Finally, there is some evidence that deep brain stimulation may alleviate pain. ${ }^{16}$ For complicated patients with intractable pain, a multidisciplinary approach is essential, including addressing underlying depression, which is a frequent comorbidity, and implementing various exercise programs. Finally, in patients who are non-verbal, it is important to remember that pain is a frequent cause of agitation that should be assessed with any new behavioral problems.

\section{Autonomic dysfunction}

Autonomic dysfunction in PD can either be secondary to the disease itself or a side effect of dopaminergic medications. Common symptoms include constipation, urinary urgency and frequency, orthostatic hypotension, hyperhidrosis, drooling, and sexual dysfunction. A study of 141 patients with PD and 50 healthy age-matched controls showed that the prevalence of these symptoms was significantly higher than in controls, and $50 \%$ of patients with PD rated their effect on daily living as "a lot" or "very much."17

Management of autonomic dysfunction in PD can be complex. The first step should be to adjust dopaminergic therapies when feasible. ${ }^{18}$ Orthostatic hypotension, for instance, is a common symptom with treatments that vary widely in burdensomeness. Less invasive measures include counter maneuvers (leg crossing, toe raising, and thigh contraction), elevating the head of bed, wearing elastic stockings and/or abdominal binders, and exercising. ${ }^{19}$ If these measures are ineffective, patients can be asked to increase fluid and salt intake, and potentially start on medications, such as fludrocortisone and midodrine. For urinary dysfunction, treatments can span from noninvasive management, such as incontinence briefs and bedside commodes, to pelvic floor physical therapy and percutaneous posterior tibial nerve stimulation(PTNS). ${ }^{20}$ More invasive treatments can include anticholinergic medications, which come with undesirable side effects, such as dizziness, blurred vision, confusion, and constipation, and botulinum toxin injections in the detrusor muscle. ${ }^{21}$ Drooling is another nonmotor complaint, that is, of significant distress to PD patients due to the social stigma. Given that the drooling is not due to excess saliva production but rather dysphagia causing saliva accumulation, less invasive treatments include speech therapy and tactics to encourage swallowing, such as chewing on gum or sucking on hard candy. Medication, such as glycopyrrolate, scopolamine, and trihexyphenidyl, can be used to reduce saliva formation, but can also thicken saliva making it more difficult to swallow. Furthermore, these medications come with a multitude of bowel and bladder as well as cognitive side effects. Atropine eye drops can be given locally under the tongue to limit systemic side effects. ${ }^{22}$ In severe cases, botulinum injections into salivary glands can be considered. ${ }^{23}$

\section{Mood disorders}

Prominent mood disorders in PD include depression, anxiety, apathy, and psychosis, which can negatively impact quality of life for both patients and their caregivers by affecting sleep, worsening fatigue, and limiting socialization. ${ }^{24,25}$ Reported prevalence is quite variable; depression rates range between $2.7 \%$ and $>90 \%,{ }^{26}$ anxiety rates range between $20 \%$ and $49 \%,{ }^{27,28}$ apathy rates average $\sim 38 \%,{ }^{29}$ and psychosis rates range between $5 \%$ and $72 \%$ dependent on hallucination type. $^{30}$

Studies have suggested that some of the mood issues are associated with "off" periods of PD, warranting adjustment of dopaminergic medications to minimize these periods..$^{31,32}$ Pramipexole, in particular, has been compared to fluoxetine and sertraline for depression treatment, and was found to be superior to both serotonergic medications. ${ }^{33,34}$ It is also suggested that anxiety, which often coincides with depression in PD, improves with dopaminergic intervention to stabilize motor symptoms and reduces the duration of "off" periods, which can at times manifest as "panic attacks." "35

Regarding apathy, there are a few studies suggesting symptom improvement with dopaminergic medications. ${ }^{36}$ Lastly, psychotic symptoms, including auditory and visual hallucinations, have been attributed with side effects of typical 
dopaminergic PD medications, and can improve with dosing adjustments. ${ }^{37}$

If there is no improvement with adjustment of dopaminergic medications, nondopaminergic medications can be considered for treatment of mood disorders. Despite less evidence associated with serotonergic medications and mixed serotonin/norepinephrine reuptake inhibitors, such as sertraline, citalopram, fluoxetine, and venlafaxine, they can be useful for depression and anxiety management and are generally well tolerated. ${ }^{38}$ For psychosis, atypical antipsychotic medications, such as clozapine, risperidone, olanzapine, quetiapine, ziprasidone, and aripiprazole, can be used, though judiciously, as they can also worsen a patient's motor symptoms. As to which antipsychotic medication is more efficacious in PD patients, the literature is thus far inconclusive.

As with the other nonmotor symptoms, if the patient's mood disorder cannot be improved with adjustments in the PD medication regimen or if the side effect burden is too great with the addition of new medications, nonpharmacologic interventions can also be considered. Structured psychological interventions, including cognitive behavioral therapy, supportive therapy, and psychoeducation, can be utilized..$^{39,40}$ In addition, for hallucinations in less impaired PD patients, self-driven coping strategies, such as cognitive, interactive, and visual techniques, can sometimes be adequately effective. ${ }^{41}$ Such strategies include turning on the lights, self-reassurance that the hallucination will resolve shortly, engaging caregivers to further gain reassurance and comfort, looking away from the direction of the hallucination, and focusing on a different object.

\section{Sleep disorders}

Sleep disorders are prominent in PD patients, and are often considered a prodrome to the development of PD motor symptoms. A study by Schenck et al in 2013 showed that $38 \%$ of patients with REM sleep behavior disorder (RBD) developed PD after 5-year follow-up. ${ }^{42}$ After PD diagnosis, sleep disturbances remain a significant issue for patients, with reported incidence of $\sim 60 \%-70 \%$. It is thought that the disturbances are either associated with the disease itself or due to side effects of PD medications. ${ }^{43}$ Common complaints include difficulties falling or staying asleep, daytime sleepiness with sleep attacks, sleep fragmentation, and violent dreams and dream enactment behavior consistent with RBD. A study by Duncan et al showed that sleep disturbances have a significant impact on health-related quality of life assessments warranting intervention. ${ }^{44}$
Interventions are largely dependent on the underlying etiology of the patient's sleep disturbance..$^{45}$ For instance, if a patient complains of insomnia, possible etiologies to consider in addition to natural disease progression include side effects associated with the patient's PD medications, and concurrent nonmotor symptoms, such as pain, urinary issues, depression, hallucinations, and psychosis that can disrupt sleep. PD medications can be adjusted or timed differently to optimize motor symptoms control and minimize side effects. Management of the nonmotor symptoms as discussed earlier can be helpful as well, though it is important to be aware that some of the interventions can actually further disrupt sleep. For example, serotonergic medications for depression can contribute to sleep disturbances. Nonpharmacologic methods to improve sleep hygiene can be beneficial, including limiting daytime naps, cognitive behavioral therapy, and relaxation training. ${ }^{46}$ Melatonin $(5-50 \mathrm{mg}$ ) can also be utilized to normalize circadian rhythm. ${ }^{47}$ Another common sleep disturbance is RBD, which as previously mentioned can often precede the development of PD motor symptoms. Clonazepam $(0.5-1.0 \mathrm{mg})$ has been reported to be helpful for RBD treatment, with a response rate averaging $80 \%-90 \%{ }^{48}$ Melatonin (3-12 $\mathrm{mg}$ ) can be used as well, in particular, if benzodiazepines are contraindicated due to other comorbidities. ${ }^{49}$

\section{Medication burden}

Patients with late stage PD often have multiple medical comorbidities given the typical age of onset. ${ }^{50,51}$ In addition to the medications prescribed for motor symptoms, patients are often taking medications for nonmotor symptoms, and other non-neurologic diseases. Polypharmacy can become quite a problem, creating undesirable side effects and drug interactions, affecting quality of life due to frequency of medication administration, increasing aspiration risks due to pill swallowing, and exacerbating financial burden due to medication costs. ${ }^{52}$ It can also affect caregiver burden as well, given the frequency of drug administration..$^{53}$ Thus, consciously simplifying/streamlining the patient's medication regimen and treating symptoms with nonpharmacologic means is desirable.

\section{Caregiver}

Emphasis on caregiver needs is of particular importance given that caregiver physical and emotional health is especially vulnerable in late stage disease and that patients' quality of life is heavily dependent on their caregivers' health and well-being. ${ }^{54-58}$ Thus, equal attention needs to be paid for 
addressing caregiver needs in the palliative care setting and this is really only possible with an interdisciplinary approach.

The caregiver, who plays a pivotal role in the patient's health care, is often viewed by health care professionals as part of the health care delivery system rather than a recipient of care. ${ }^{59-61}$ In palliative care, the caregivers are considered equal recipients of care and receive an independent assessment regarding their needs. Four areas of assessment help define the plan of care: 1) caregiver strain, 2) capacity and strengths of the caregiver, 3) preparedness/knowledge base, and 4) care needs of the patient.

\section{Caregiver strain}

Strain is defined as the perceived difficulty in fulfilling the caregiving role. Strain increases with disease severity and can lead to a decline in the physical and emotional health of the caregiver. ${ }^{3}$ The first step for managing caregiver strain is to measure the strain severity. This can be done through standardized caregiver strain scales. Although no one scale is universally used, many have been helpful as screening tools in clinical practice. For instance, the MCSI has been used across many PD centers. ${ }^{62}$ It has six subscales, consisting of physical strain, social constraints, financial strain, time constraints, interpersonal strain, and patient demanding/ manipulative behavior. A cutoff value of $\geq 30$ indicates severe strain. ${ }^{63}$

\section{Caregiver capacity}

Certain caregiver characteristics can directly influence caregiving capacity. ${ }^{64}$ Specifically, female gender, young age, poor emotional and physical health, low economic status, rural living situation, employment outside of the home, number of hours/day providing direct care, poor self-care behaviors, and limited social support predict strain. Interventions to strengthen caregiving capacity may include improving caregiver health and social support. ${ }^{58}$ At the same time, it is vitally important that health professionals take the time to identify and praise caregivers' work and the positive aspects and value that they may derive from their service. Thanking caregivers for the good things that they are doing can make a huge difference to their self-esteem, well-being, and ability to continue. ${ }^{65,66}$

\section{Preparedness/knowledge}

A checklist of knowledge needs can be helpful to maximize caregiver preparedness and minimize strain. This checklist may include the following: lifting and moving skills, medication management, symptom management, communication skills, problem-solving skills, community resources (e.g., support groups), advanced care planning, and respite care. ${ }^{67}$

\section{Patient needs}

Caregiver strain can be directly affected by the type and extent of patient care needs. Studies have shown that severity of PD symptoms and frequency of falls can significantly contribute to the degree of caregiver burden. ${ }^{68,69}$ Likewise, neuropsychiatric symptoms, such as anxiety, depression, apathy, and psychosis, in conjunction with dementia can increase caregiver burden and can lead to institutionalization of PD patients. ${ }^{56,58,64}$

Overall, a systematic caregiver assessment can help provide direction on how to best help the family. Although caregiver intervention research is still in its infancy, there is some evidence that psychoeducational programs can be beneficial. ${ }^{70-74}$ These programs not only provide education, but also teach caregivers skills and self-efficacy. One example is the Stanford Chronic Disease Self-Management Program. It provides educational content and teaches participants skills, such as problem solving, decision making, and communication. ${ }^{75}$ In Oregon, this program is called Strive to Thrive and is offered specifically for people with PD and their caregivers.

\section{Physical therapy}

Typically, the role of physical therapy in PD patient care is to maximize independence and safety revolving around the patient's mobility. In the interdisciplinary clinic, this role extends more broadly to include quality of life concerns and symptoms, such as incontinence and pain.

A significant concern of patients and families in the later stages of PD is worsening mobility and increasing falls, which are often the result of gait freezing, postural instability, and gait disturbances. The PT uses gait, balance, and muscle strength exams to determine the appropriate assistive device to best maintain a patient's independence and safety. Currently, there are several different types of walkers on the market, including the four-wheeled walker, front-wheeled walker, and U-step walker. A study by Kegelmeyer et al ${ }^{76}$ compared the different walkers utilizing quantitative gait measures, including velocity and stride length. The four-wheeled walker was shown to have less variability in gait measures, and less impact on spontaneous unassisted gait patterns. As each PD patient is unique, it is often beneficial to trial a variety of walkers to determine the most suitable option. For instance, patients with gait freezing may prefer a U-step walker because of its increased stability and the option to mount a laser and metronome that can help with step initiation. In addition, a 
PT can provide recommendations on wheelchairs that provide appropriate postural support for either forward or lateral lean tendencies, and also provide pressure-unloading cushions to prevent skin breakdown. Similar to walkers, there are several different types of wheelchairs (power, tilt-in-space, manual) that can be individualized to maximize patient comfort and mobility.

In addition to assistive devices, several prosthetics have been developed for protection from falls, including hip protectors. Thus far, there is limited evidence on the efficacy of hip protectors in preventing hip fractures, ${ }^{77}$ in part due to the relatively low compliance in wearing the devices. ${ }^{78}$ The evidence, thus far, best supports the use of hip protectors in nursing homes and residential care settings over community dwellers. Clinically, we have found that external protectors (e.g., SafeHip Active) are more acceptable to patients than the traditional underwear model due to improved ease of donning/doffing and reported improved comfort.

Urinary dysfunction, such as urgency, increased daytime frequency, nocturia, and incontinence, is the most common clinical manifestation of autonomic dysfunction in $\mathrm{PD}$, with $\sim 70 \%$ of patients developing symptoms within 5 years of diagnosis. ${ }^{79}$ Unfortunately, urinary symptoms, such as incontinence and frequency, can lead to falls and impact quality of life..$^{80,81}$ As discussed in the "Neurology clinician" section, medications can be prescribed for urinary dysfunction, but often come with undesirable side effects and can contribute to polypharmacy. Percutaneous PTNS is a minimally invasive neuromodulation technique used to improve urinary symptoms that can be done either in the outpatient setting by a PT or a urologist, or in the home with the purchase of a neuromuscular electrical stimulation unit. Kabay et $\mathrm{l}^{82}$ recently demonstrated that 12 weeks of PTNS treatment improved urinary symptoms in PD patients, namely incontinence and frequency. There is also some evidence that pelvic floor physical therapy consisting of behavioral therapy and electromyography biofeedback for pelvic floor control can improve quality of life measures and reduce urinary incontinence frequency. ${ }^{83}$ Other supportive devices, such as a bedside commode, condom catheter, bidet, and appropriate incontinence garments, can improve both patient quality of life and caregiver strain. Patients and their caregivers can often benefit from a one-time consultation with either an occupational therapist or pelvic floor PT to go over practical tips for behavioral and lifestyle modifications (e.g., transfer training, bladder diary, and elevation of legs prior to bed).

As outlined in the "Neurology clinician" section, pain is a major concern for many patients with PD and it can be difficult to treat medically depending on the etiology of the pain. The role of the PT is to help determine an appropriate exercise program to alleviate symptoms. ${ }^{84}$ Specifically, dystonic pain has anecdotally been shown to improve with sensory trick or bracing, which changes the sensory input to inhibit the dystonic muscles. For instance, an example of a sensory trick for dystonia localized to the toe involves taping a penny to the toe itself for added weight and change in sensory input. For bracing, toe socks, spreaders, and supra malleolar orthosis have been used to improve foot dystonia. Again, these are anecdotal reports of improvement in symptoms; to date there is no published data regarding the evidence behind these techniques. Regarding rigidity, it has been shown to respond well to stretching and manual therapy to treat potential musculoskeletal restrictions. Again, the published data on $\mathrm{PD}$ rigidity and physical therapy are limited; there is no unified consensus on what types of exercises are more effective.

\section{Speech therapy}

PD patients present with complex communicative challenges that evolve and change over time. These challenges can impact the domains of speech, language, and cognition, each of which may need addressing at different stages in the disease process. In the Next Step Clinic, the SLP evaluates the patient's communication, cognition, and swallowing abilities to assess for areas of deficit and challenges to daily function. Depending upon the priorities of the patient and family, recommendations are made for further evaluation and treatment.

In late stage $\mathrm{PD}$, the patient often has already completed voice therapy or Lee Silverman Voice Treatment (LSVT) ${ }^{85}$ to treat hypophonia or hypokinetic dysarthria, but continues to struggle with day-to-day communication. Some patients can benefit from a refresher of LSVT, whereas others may need supported communication in the form of amplification, amplified telephone, or low/high tech communication systems that may or may not be combined with verbal communication.

Despite voice therapy and communication devices, patients can still have significant communication difficulties due to cognitive impairment. Although the presence and extent of cognitive impairment vary from patient to patient, $\sim 27 \%$ may experience at least mild cognitive impairment and $\sim 40 \%$ may eventually develop dementia. ${ }^{86,87}$ Risk furthermore increases with age and disease duration, ${ }^{88}$ and increases burden on the patient, caregiver, and community. ${ }^{89}$ Patients with cognitive impairments may find difficulty holding and maintaining conversations, resulting in decreased participation and social isolation. Techniques can be taught by the SLP to both the patient and caregiver to increase participation. 
For patients with difficulty keeping up with conversations, "speaker control" strategies, such as making eye contact with the speaker, asking the speaker to repeat if necessary, asking the speaker to slow down or speak up, and asking the speaker to give one piece of information at a time, can be utilized to keep up with the conversation. Caregivers may be taught strategies to invite patients into conversation when they begin to withdraw.

It is important to emphasize that regardless of how impaired communication may be, there will always be a way to communicate on some level as long as the patient is willing. This is of particular significance in the context of a debilitating disease such as PD, which places patients at increased risk for social isolation due to physical, cognitive, and communicative challenges. Palmer et $\mathrm{al}^{90}$ analyzed data from a representative national sample of community-dwelling adults aged $\geq 65$ years and found that communication difficulty was predictive of smaller social network size, fewer positive social exchanges, less frequent participation in social activities, and higher levels of loneliness. Holt-Lunstad et $\mathrm{al}^{91}$ concluded that "individuals with adequate social relationships have a 50\% greater likelihood of survival compared to those with poor or insufficient social relationships." Therefore, maximizing communication capabilities is an essential part of maintaining health at every stage of PD.

Aside from assisting with speech and communication interventions, SLP also plays a significant role in managing dysphagia common to PD patients. Swallowing problems can increase risk for aspiration, which can lead to pneumonia and possibly death. These problems are often identified in clinic, and the patient is then referred for a more objective evaluation and intervention. Patient and family are educated on aspiration precautions, dietary modifications, and techniques to maximize swallowing safety as indicated. The direction or extent of intervention may coincide with a discussion on goals of care, including whether the patient would want a feeding tube, such as a percutaneous endoscopic gastrostomy (PEG) tube, placed or wish to be intubated should he or she develop acute respiratory distress from an aspiration pneumonia. Importantly, PEG tube placement is not recommended for all patients, and should only be pursued after careful consideration of the patient's wishes, explanation of risks and benefits, and emphasis that it may not prolong life. ${ }^{92,93}$ The SLP can play a significant role in this discussion by illustrating daily life with a feeding tube to the patient and family. Given the gravity of such conversation and focus on goals of care, the discussion may ultimately lead to the completion of an Advanced Directive with the guidance of the neurology clinician and/or SW.

\section{Social work}

The role of the SW in the care of late stage PD patients can be quite extensive, often warranting several meetings outside of clinic, sometimes at the home. The SW, by being part of the interdisciplinary clinic, has the opportunity to begin developing the narrative, that is, so important to future counseling, especially with regards to palliative care issues. Such issues include caregiver strain/capacity, advanced care planning, and planning for loss of function and decision-making capacity. The client involved can encompass the patient, caregiver, and family unit.

Counseling around emotional, spiritual, and existential pain is an important part of social work in late stage PD. ${ }^{94,95}$ This counseling is more cognitive, behavioral, humanistic, and short to medium term than psychodynamic and long term. If the client is amenable, narrative therapy work can extend to such practices as solution focused therapy, motivational interviewing, cognitive behavioral therapy, ${ }^{39,40}$ behavioral activation, Gottman couples therapy, ${ }^{96}$ acceptance and commitment therapy, mindfulness practice, ${ }^{97}$ etc.

Though palliative care tends to focus on transitions, there is frequently a crisis that demands near term counseling and solutions. Crises tend to involve changes in physical, functional, or cognitive capacity that create anxiety around managing a life in the present and planning a life in the future. Collecting and completing documentation of future plans can help manage this anxiety and allow the client to put more thought toward life activities that create meaningful memories. Assessment of future plans may include the choices involved in: Financial Power of Attorney, Advance Directive for Healthcare, Physician Orders for Life Sustaining Treatment, Social Security Retirement Benefits, Social Security Disability Insurance, Medicaid, Medicare, Veterans' Benefits, Family Medical Leave Act, reasonable accommodations in employment under The Americans with Disabilities Act, and many state, local and community resources. There are a great number and variety of state, local, and community resources accessible to PD patients, and SWs are frequently the most knowledgeable about these resources. Review of advance care planning documents, especially when facilitated by the SW, can help patients and their families start an ongoing conversation about goals, fears, and preferences while the patient is still able to be an active participant and before a crisis.

Social work fits well in the comprehensive model of interdisciplinary care for late stage PD as a short-term counselor and also a guide to resources. The overall focus of counseling is socialization for both the patient and the 
caregiver. Research in interpersonal neurobiology suggests that storytelling, secure attachment, emotional regulation, and knowledge of learning style (i.e., what things are fun) are the benefits of socialization and support a healthy mind. ${ }^{98}$ Anxiety, depression, and apathy increase with poor socialization, and severely impact quality of life. ${ }^{99}$ Socialization is also part of the narrative with family, who may step in as respite caregivers and create social opportunities for both the patient and caregiver. The SW may help facilitate such socialization by helping the client work through existing family systems, attachment issues or past traumas. Lastly, the SW serves as a guide to local resources, which importantly include PD support groups. Studies have shown that PD patients who attend such support groups have improved quality of life, fewer depression and anxiety symptoms, and less social phobia. ${ }^{100}$

\section{Nursing}

An interdisciplinary care clinic can be challenging to coordinate and run efficiently in order to maximize providers' time and expertise to address patient and family goals for care. The nurse is the primary case manager or care coordinator for the clinic and ensures that the recommended treatment plan is implemented to the benefit of the patient and family. Nurses are trained to fulfill several roles in the care of a patient, including clinical care coach, team coordinator, counselor, educator, advocate, and medical provider liaison, which make them the ideal person to fulfill this role.

For the Next Step Clinic, the nurse works to coordinate referrals, staff assignments, information triage, and health care plan implementation. This includes communicating with referring providers on appropriateness of referrals, scheduling, and communicating to the patient and caregiver(s) the goals of the interdisciplinary clinic. On the actual clinic day, the nurse organizes and initiates the opening care conference, followed by the multiple team meetings throughout the course of the day. Given that multiple patients are seen by multiple team members within a limited time frame, the nurse helps to maintain the flow of the clinic and keeps the team members on track with time. After the final end-of-visit treatment plan has been discussed with the patient and family, the nurse's role continues with finalizing and carrying out the recommendations utilizing a specialized and limited protocol to facilitate orders for referrals. The nurse also researches healthcare resources nearby the patient and caregiver's home such as home health services. Finally, the nurse serves as a liaison with patient and caregiver for follow-up implementation, education, and care plan compliance. Overall, because of the nurse's experience and expertise in PD care planning and treatment, and understanding of each team discipline as they relate to the various progressive stages of $\mathrm{PD}$, the nurse plays a crucial role in the management, operation, and execution of the palliative, interdisciplinary care clinic.

\section{Conclusion}

Interdisciplinary palliative care for late stage PD has been a growing field of interest in the recent years. Compared to ALS, where well-designed studies have shown increased survival with multidisciplinary/interdisciplinary care, the data in the PD population have thus far been limited. ${ }^{101,102}$ As illustrated earlier, the complexity of care for late stage PD patients in terms of severity and extent of both motor and nonmotor symptoms, increasing caregiver burden, and impending discussions of goals of care warrant a team approach to meeting the needs of both patient and caregiver(s). Further studies will be essential to assessing the impact of this approach to patient outcomes in PD.

\section{Disclosure}

The authors report no conflicts of interest in this work.

\section{References}

1. Huse DM, Schulman K, Orsini L, Castelli-Haley J, Kennedy S, Lenhart G. Burden of illness in Parkinson's disease. Mov Disord. 2005; 20(11):1449-1454

2. Higginson IJ, Gao W, Saleem TZ, et al. Symptoms and quality of life in late stage parkinson syndromes: a longitudinal community study of predictive factors. PLoS One. 2012;7(11):e46327.

3. Carter JH, Stewart BJ, Archbold PG, et al. Living with a person who has parkinson's disease: the spouse's perspective by stage of disease. Parkinson's Study Group. Mov Disord. 1998;13(1):20-28.

4. WHO Definition of Palliative Care. Available from: http://www.who. int/cancer/palliative/definition. Accessed January 5, 2017.

5. Traynor BJ, Alexander M, Corr B, Frost E, Hardiman O. Effect of a multidisciplinary amyotrophic lateral sclerosis (ALS) clinic on ALS survival: a population based study, 1996-2000. J Neurol Neurosurg Psychiatry. 2003;74(9):1258-1261.

6. Chaudhuri KR, Odin P, Antonini A, Martinez-Martine P. Parkinson's disease: the non-motor issues. Parkinsonism Relat Disord. 2011; 17(10):717-723.

7. Poewe W. Non-motor symptoms in Parkinson's disease. Eur J Neurol. 2008;15(1):14-20.

8. Seppi K, Weintraub D, Coelho M, et al. The movement disorder society evidence-based medicine review update: treatments for the non-motor symptoms of Parkinson's disease. Mov Disord. 2011;26(Suppl 3): S42-S80.

9. Beiske AG, Loge JH, Rønningen A, Svensson E. Pain in parkinson's disease: prevalence and characteristics. Pain. 2009;141(1-2): 173-177.

10. Defazio G, Berardelli A, Fabbrini G, et al. Pain as a nonmotor symptom of parkinson disease: Evidence from a case-control study. Arch Neurol. 2008;65(9):1191-1194.

11. Lee MA, Walker RW, Hildreth TJ, Prentice WM. A survey of pain in idiopathic parkinson's disease. J Pain Symptom Manage. 2006;32(5): $462-469$. 
12. Politis M, Wu K, Molloy S, G Bain P, Chaudhuri KR, Piccini P. Parkinson's disease symptoms: the patient's perspective. Mov Disord. 2010;25(11):1646-1651.

13. Roh JH, Kim BJ, Jang JH, et al. The relationship of pain and healthrelated quality of life in Korean patients with Parkinson's disease. Acta Neurol Scand. 2009;119(6):397-403.

14. Goy ER, Carter J, Ganzini L. Neurologic disease at the end of life: caregiver descriptions of parkinson disease and amyotrophic lateral sclerosis. J Palliat Med. 2008;11(4):548-554.

15. Ford B. Pain in Parkinson's disease. Mov Disord. 2010;25:S98-S103.

16. Cury RG, Galhardoni R, Fonoff ET, et al. Effects of deep brain stimulation on pain and other nonmotor symptoms in Parkinson disease. Neurology. 2014;83(16):1403-1409.

17. Magerkurth C, Schnitzer R, Braune S. Symptoms of autonomic failure in Parkinson's disease: prevalence and impact on daily life. Clin Auton Res. 2005;15(2):76-82.

18. Pfeiffer RF. Autonomic dysfunction in Parkinson's disease. Expert Rev Neurother. 2012;12(6):697-706.

19. Mostile G, Jankovic J. Treatment of dysautonomia associated with Parkinson's disease. Parkinsonism Relat Disord. 2009;15(Suppl 3):S224-S232.

20. Kabay SC, Kabay S, Yucel M, Ozden H. Acute urodynamic effects of percutaneous posterior tibial nerve stimulation on neurogenic detrusor overactivity in patients with Parkinson's disease. Neurourol Urodyn. 2009;28(1):62-67.

21. Giannantoni A, Rossi A, Mearini E, Del Zingaro M, Porena M, Berardelli A. Botulinum toxin A for overactive bladder and detrusor muscle overactivity in patients with Parkinson's disease and multiple system atrophy. J Urol. 2009;182(4):1453-1457.

22. Hyson HC, Johnson AM, Jog MS. Sublingual atropine for sialorrhea secondary to parkinsonism: a pilot study. Mov Disord. 2002;17(6): 1318-1320.

23. Friedman A, Potulska A. Quantitative assessment of parkinsonian sialorrhea and results of treatment with botulinum toxin. Parkinsonism Relat Disord. 2001;7(4):329-332

24. McKinlay A, Grace RC, Dalrymple-Alford JC, Anderson T, Fink J, Roger D. A profile of neuropsychiatric problems and their relationship to quality of life for Parkinson's disease patients without dementia. Parkinsonism Relat Disord. 2008;14(1):37-42.

25. Schrag A, Jahanshahi M, Quinn N. What contributes to quality of life in patients with Parkinson's disease? J Neurol Neurosurg Psychiatry. 2000;69(3):308-312.

26. Reijnders JS, Ehrt U, Weber WE, Aarsland D, Leentjens AF. A systematic review of prevalence studies of depression in Parkinson's disease. Mov Disord. 2008;23(2):183-189.

27. Gallagher DA, Schrag A. Psychosis, apathy, depression and anxiety in Parkinson's disease. Neurobiol Dis. 2012;46(3):581-589.

28. Pontone GM, Williams JR, Anderson KE, et al. Prevalence of anxiety disorders and anxiety subtypes in patients with Parkinson's disease. Mov Disord. 2009;24(9):1333-1338.

29. Pedersen KF, Larsen JP, Alves G, Aarsland D. Prevalence and clinical correlates of apathy in Parkinson's disease: A community-based study. Parkinsonism Relat Disord. 2009;15(4):295-299.

30. Fénelon G, Alves G. Epidemiology of psychosis in Parkinson's disease. J Neurol Sci. 2010;289(1-2):12-17.

31. Maricle RA, Nutt JG, Valentine RJ, Carter JH. Dose-response relationship of levodopa with mood and anxiety in fluctuating Parkinson's disease: a double-blind, placebo-controlled study. Neurology. 1995;45(9):1757-1760.

32. Menza MA, Sage J, Marshall E, Cody R, Duvoisin R. Mood changes and "on-off" phenomena in Parkinson's disease. Mov Disord. 1990;5(2):148-151.

33. Barone P, Scarzella L, Marconi R, et al; Depression/Parkinson Italian Study Group. Pramipexole versus sertraline in the treatment of depression in Parkinson's disease. J Neurol. 2006;253(5):601-607.

34. Goldberg JF, Burdick KE, Endick CJ. Preliminary randomized, double-blind, placebo-controlled trial of pramipexole added to mood stabilizers for treatment-resistant bipolar depression. Am J Psychiatry. 2004;161(3):564-566.
35. Richard IH, Schiffer RB, Kurlan R. Anxiety and Parkinson's disease. J Neuropsychiatry Clin Neurosci. 1995;8(4):383-392.

36. Czernecki V, Schüpbach M, Yaici S, et al. Apathy following subthalamic stimulation in Parkinson disease: a dopamine responsive symptom. Mov. Disord. 2008;23(7):964-969.

37. Kuzuhara S. Drug-induced psychotic symptoms in Parkinson's disease: problems, management and dilemma. J Neurol. 2001;248(Suppl 3): 11128-11131.

38. Schrag A. Psychiatric aspects of Parkinson's disease. J Neurol. 2004;251(7):795-804.

39. Dobkin RD, Menza M, Allen LA, et al. Cognitive-behavioral therapy for depression in Parkinson's disease: a randomized, controlled trial. Am J Psychiatry. 2011;168(10):1066-1074.

40. Cole K, Vaughan FL. The feasibility of using cognitive behaviour therapy for depression associated with Parkinson's disease: a literature review. Parkinsonism Relat Disord. 2005;11(5):269-276.

41. Diederich NJ, Pieri V, Goetz CG. Coping strategies for visual hallucinations in Parkinson's disease. Mov Disord. 2003;18(7):831-832.

42. Schenck CH, Boeve BF, Mahowald MW. Delayed emergence of a parkinsonian disorder or dementia in $81 \%$ of older men initially diagnosed with idiopathic rapid eye movement sleep behavior disorder: a 16-year update on a previously reported series. Sleep Med. 2013;14(8):744-748.

43. Oerlemans WG, de Weerd AW. The prevalence of sleep disorders in patients with Parkinson's disease: a self-reported, community-based survey. Sleep Med. 2002;3(2):147-149.

44. Duncan GW, Khoo TK, Yarnall AJ, et al. Health-related quality of life in early Parkinson's disease: the impact of nonmotor symptoms. Mov Disord. 2014;29(2):195-202.

45. Schrempf W, Brandt MD, Storch A, Reichmann H. Sleep disorders in parkinson's disease. J Parkinsons Dis. 2014;4(2):211-221.

46. Friedman JH, Chou KL. Sleep and fatigue in Parkinson's disease. Parkinsonism Relat Disord. 2004;10(Suppl 1):S27-S35.

47. Dowling GA, Mastick J, Colling E, Carter JH, Singer CM, Aminoff MJ. Melatonin for sleep disturbances in Parkinson's disease. Sleep Med. 2005;6(5):459-466.

48. Tan A, Salgado M, Fahn S. Rapid eye movement sleep behavior disorder preceding Parkinson's disease with therapeutic response to levodopa. Mov Disord. 1996;11(2):214-216.

49. Boeve BF, Silber MH, Ferman TJ. Melatonin for treatment of REM sleep behavior disorder in neurologic disorders: results in 14 patients. Sleep Med. 2003;4(4):281-284.

50. Pohar SL, Allyson Jones C. The burden of Parkinson disease (PD) and concomitant comorbidities. Arch Gerontol Geriatr. 2009;49(2): 317-321.

51. Lubomski M, Rushworth RL, Tisch S. Hospitalisation and comorbidities in Parkinson's disease: a large Australian retrospective study. J Neurol Neurosurg Psychiatry. 2015;86(3):324-330.

52. Bainbridge JL, Ruscin JM. Challenges of treatment adherence in older patients with parkinson's disease. Drugs Aging. 2009;26(2):145-155.

53. Ranelli PL, Aversa SL. Medication-related stressors among family caregivers. Am J Health Syst Pharm. 1994;51(1):75-79.

54. Terriff DL, Williams JV, Patten SB, Lavorato DH, Bulloch AG. Patterns of disability, care needs, and quality of life of people with Parkinson's disease in a general population sample. Parkinsonism Relat Disord. 2012;18(7):828-832.

55. Hassan A, Wu S, Schmidt P, et al. What are the issues facing Parkinson's disease patients at ten years of disease and beyond? Data from the NPF-QII study. Parkinsonism Relat Disord. 2012;18(Suppl 3): S10-S14.

56. Martinez-Martin P, Rodriguez-Blazquez C, Forjaz MJ, et al. Neuropsychiatric symptoms and caregiver's burden in Parkinson's disease. Parkinsonism Relat Disord. 2015;21(6):629-634.

57. Miyasaki J, Kluger B. Palliative care for parkinson's disease: has the time come? Curr Neurol Neurosci Rep. 2015;15(5):26.

58. Greenwell K, Gray WK, van Wersch A, van Schaik P, Walker R. Predictors of the psychosocial impact of being a carer of people living with Parkinson's disease: a systematic review. Parkinsonism Relat Disord. 2015;21(1):1-11. 
59. Lageman SK, Mickens MN, Cash TV. Caregiver-identified needs and barriers to care in Parkinson's disease. Geriatr Nurs. 2015; 36(3):197-201.

60. Richfield EW, Jones EJ, Alty JE. Palliative care for Parkinson's disease: A summary of the evidence and future directions. Palliat Med 2013;27(9):805-810.

61. Hudson P, Payne S. Family caregivers and palliative care: current status and agenda for the future. J Palliat Med. 2010;14(7):864-869.

62. Oguh O, Kwasny M, Carter J, Stell B, Simuni T. Caregiver strain in Parkinson's disease: national parkinson foundation quality initiative study. Parkinsonism Relat Disord. 2013;19(11):975-979.

63. Stull DE. The multidimensional caregiver strain index (MCSI): its measurement and structure. J Clin Geropsychol. 1996;2(3):175-96.

64. Lyons KS, Stewart BJ, Archbold PG. Optimism, Pessimism, Mutuality, and Gender: Predicting 10-Year Role Strain in Parkinson's Disease Spouses. Gerontologist. 2009;49(3):378-387.

65. Levine C. The Loneliness of the Long-Term Care Giver. NEngl J Med. 1999;340(20):1587-1590.

66. Lloyd J, Patterson T, Muers J. The positive aspects of caregiving in dementia: a critical review of the qualitative literature. Dementia. 2016;15(6):1534-1561.

67. Given B, Sherwood PR, Given CW. What Knowledge and Skills do caregivers need? Am J Nurs. 2008;108(Suppl 9):28-34.

68. D'Amelio M, Terruso V, Palmeri B, et al. Predictors of caregiver burden in partners of patients with Parkinson's disease. Neurol Sci. 2009;30(2):171-174.

69. Schrag A, Hovris A, Morley D, Quinn N, Jahanshahi M. Caregiverburden in parkinson's disease is closely associated with psychiatric symptoms, falls, and disability. Parkinsonism Relat Disord. 2006;12(1):35-41.

70. Hudson P, Remedios C, Zordan R, et al. Guidelines for the psychosocial and bereavement support of family caregivers of palliative care patients. J Palliat Med. 2012;15(6):696-702.

71. Hudson P, Aranda S. The Melbourne family support program: evidence-based strategies that prepare family caregivers for supporting palliative care patients. BMJ Support Palliat Care. 2014;4(3):231-237.

72. Hudson P, Trauer T, Kelly B, et al. Reducing the psychological distress of family caregivers of home based palliative care patients: longer term effects from a randomised controlled trial. PsychoOncology 2015;24(1):19-24.

73. Corry M, While A, Neenan K, Smith V. A systematic review of systematic reviews on interventions for caregivers of people with chronic conditions. J Adv Nurs. 2015;71(4):718-734.

74. Volicer L, Simard J. Palliative care and quality of life for people with dementia: medical and psychosocial interventions. Int Psychogeriatr. 2015;27(10):1623-1634.

75. Lorig KR, Ritter P, Stewart AL, et al. Chronic disease self-management program: 2-year health status and health care utilization outcomes. Med care. 2001;39(11):1217-1223

76. Kegelmeyer DA, Parthasarathy S, Kostyk SK, White SE, Kloos AD. Assistive devices alter gait patterns in Parkinson disease: Advantages of the four-wheeled walker. Gait Posture. 2013;38(1):20-24.

77. Santesso N, Carrasco-Labra A, Brigardello-Peterson R. Hip protectors for preventing hip fractures in older people. Cochrane Database Syst Rev. 2014;(3):CD001255.

78. Huang HC, Lee $\mathrm{CH}, \mathrm{Wu}$ SL. Hip protectors: a pilot study of older people in Taiwan. J Clin Nurs. 2006;15(4):436-443.

79. Sakakibara R, Uchiyama T, Yamanishi T, Shirai K, Hattori T. Bladder and bowel dysfunction in Parkinson's disease. J Neural Transm. 2008;115(3):443-460.

80. Morris V, Wagg A. Lower urinary tract symptoms, incontinence and falls in elderly people: time for an intervention study. Int J Clin Pract. 2007;61(2):320-323.
81. Sakushima K, Yamazaki S, Fukuma S, et al. Influence of urinary urgency and other urinary disturbances on falls in Parkinson's disease. J Neurol Sci. 2016;360:153-157.

82. Kabay S, Canbaz Kabay S, Cetiner M, et al. The clinical and urodynamic results of percutaneous posterior tibial Nnrve stimulation on neurogenic detrusor overactivity in patients with Parkinson's Disease. Urology. 2016;87:76-81

83. Vaughan CP, Juncos JL, Burgio KL, Goode PS, Wolf RA, Johnson TM 2nd. Behavioral therapy to treat urinary incontinence in Parkinson disease. Neurology. 2011;76(19):1631-1634.

84. Allen NE, Moloney N, van Vliet V, Canning CG. The Rationale for Exercise in the Management of Pain in Parkinson's Disease. J Parkinsons Dis. 2015;5(2):229-239.

85. Ramig LO, Sapir S, Countryman S, et al. Intensive voice treatment (LSVT®) for patients with Parkinson's disease: a 2 year follow up. J Neurol Neurosurg Psychiatry. 2001;71(4):493-498.

86. Emre M, Aarsland D, Brown R, et al. Clinical diagnostic criteria for dementia associated with Parkinson's disease. Mov Disord. 2007;22(12):1689-1707.

87. Litvan I, Aarsland D, Adler CH, et al. MDS task force on mild cognitive impairment in Parkinson's disease: Critical review of PD-MCI. Mov Disord. 2011;26(10):1814-1824.

88. Hely MA, Reid WG, Adena MA, Halliday GM, Morris JG. The Sydney multicenter study of Parkinson's disease: the inevitability of dementia at 20 years. Mov Disord. 2008;23(6):837-844.

89. Aarsland D, Brønnick K, Ehrt U, et al. Neuropsychiatric symptoms in patients with Parkinson's disease and dementia: frequency, profile and associated care giver stress. J Neurol Neurosurg Psychiatry. 2007;78(1):36-42.

90. Palmer AD, Newsom JT, Rook KS. How does difficulty communicating affect the social relationships of older adults? An exploration using data from a national survey. J Commun Disord. 2016;62:131-146.

91. Holt-Lunstad J, Smith TB, Layton JB. Social relationships and mortality risk: a meta-analytic review. PLOS Med. 2010;7(7):e1000316.

92. Lokk J, Delbari A. Clinical aspects of palliative care in advanced Parkinson's disease. BMC Palliat Care. 2012;11:20.

93. Mitchell SL, Kiely DK, Lipsitz LA. The risk factors and impact on survival of feeding tube placement in nursing home residents with severe cognitive impairment. Arch Intern Med. 1997;157(3):327-332.

94. Risk JL. Building a new life: a chaplain's theory based case study of chronic illness. J Health Care Chaplain. 2013;19(3):81-98.

95. Bingham V, Habermann B. The influence of spirituality on family management of Parkinson's disease. J Neurosci Nurs. 2006;38(6):422-427.

96. Gottman JM, Krokoff LJ. Marital interaction and satisfaction: A longitudinal view. J Consult Clin Psychol. 1989;57(1):47-52.

97. Fitzpatrick Lee, Simpson J, Smith A. A qualitative analysis of mindfulness-based cognitive therapy (MBCT) in Parkinson's disease. Psychol Psychother. 2010;83(Pt 2):179-192.

98. Fishbane MD. Wired to connect: neuroscience, relationships, and therapy. Fam Process. 2007;46(3):395-412.

99. Gallagher DA, Schrag A. Psychosis, apathy, depression and anxiety in Parkinson's disease. Neurobiol Dis. 2012;46(3):581-589.

100. Artigas NR, Striebel VL, Hilbig A, et al. Evaluation of quality of life and psychological aspects of Parkinson's disease patients who participate in a support group. Dement Neuropsychol. 2015;9(3):295-300.

101. Johnston M, Chu E. Does attendance at a multidisciplinary outpatient rehabilitation program for people with Parkinson's disease produce quantitative short term or long term improvements? A systematic review. NeuroRehabilitation. 2010;26(4):375-383

102. van der Marck MA, Munneke M, Mulleners W, et al; IMPACT Study Group. Integrated multidisciplinary care in Parkinson's disease: a non-randomised, controlled trial (IMPACT). Lancet Neurol. 2013; 12(10):947-956. 
Journal of Parkinsonism and Restless Legs Syndrome is an online, open access, peer-reviewed journal. The journal publishes review articles, historical reviews, original research articles, case reports, letters to the editor, clinical teaching cases, neuroradiology highlights, neuropathology highlights, neuropsychiatry highlights, autobiographies, conference proceedings, abstracts and book reviews. The manuscript management system is completely online and includes a very quick and fair peerreview system, which is all easy to use. Visit http://www.dovepress.com/ testimonials.php to read real quotes from published authors. 